\section{GLOVES WITH A GLOSSY SHEEN}

Classic's new Pearlescent nitrile disposable examination gloves are available in pink or blue and have a unique glossy sheen. Powder-free Pearlescent gloves are ideal for sensitive hands, while providing superior barrier protection.

Not only do they eliminate Type I reactions, they are also ambidextrous and come in a luxurious pearlescent finish. Pearlescent gloves are available in a box of 100 from The Dental Directory with sizes ranging from Ex-Small to Ex-Large: gloves to suit everyone's needs. Reader response number 50

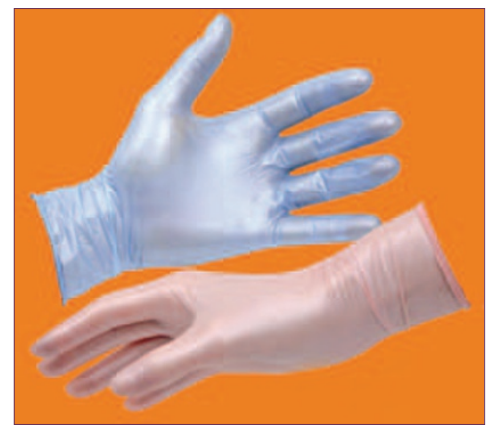

\section{ACHIEVE A NATURAL WHITE COLOUR}

Beverly Hills Formula whitening toothpaste Total Protection, Sensitive, Total Breath, Total Enamel Sensitive and Dentist's Choice: Gum \& Whitening Expert - enables patients to achieve a whiter, brighter smile in only one minute.

A study conducted at Bristol University Dental School proved that Beverly Hills Formula toothpaste can remove over $90 \%$ of staining.

These results signal a real breakthrough in oral care and aesthetics meaning your patients can restore their teeth to a natural white colour, quickly and effectively.

This impressive range of toothpastes which protects against acid attack also strengthens and re-mineralises tooth enamel for cavity protection, and is proven to be less abrasive when compared with other leading brands of both whitening and regular toothpaste. Reader response number 51

\section{CAMPAIGN FOR HEALTHY GUMS}

Gum disease is a major problem, so why are patients largely unconcerned by the symptoms? Corsodyl has recently worked with the British Dental Health Foundation (BDHF) to identify ways to motivate patients to prioritise their gum health. To help improve engagement, Corsodyl has created the 'Campaign for Healthy Gums'.

Improvements in dental care have resulted in patients retaining their natural teeth for longer, but gum disease is now the UK's leading cause of tooth loss - so why are patients largely unconcerned by the symptoms? At a recent roundtable meeting, hosted by the BDHF and supported by Corsodyl, this issue was discussed by a panel of practising dentists and hygienists.

The panel agreed that the period between dental visits is critical yet, despite oral hygiene education, patients often struggle to follow the advice provided. In addition they discussed that it is unusual for patients to approach a dental professional regarding gum problems - pain being the most likely thing to mention on a dental visit - clearly something more is needed to motivate patients to better gum health.

Subsequently the need for gum health advice which patients could easily follow outside of the dental surgery was identified.

To meet this challenge Corsodyl, a brand with over 30 years of expertise in gum care, has developed a Gum Care Guidance Pack for dental patients as part of the 'Campaign for Healthy Gums' initiative. The pack contains educational materials for use both within the waiting room and surgery as well as at home to help make it easier for patients to follow the advice provided by their dentist or hygienist.

FREE gum care packs can be ordered from GSK.

Reader response number 52

\section{THE OAMPAIGN FOR} (4)

\section{HEADS UP}

Philips has launched a new multi-pack to help dental professionals assess the benefits of each Sonicare brush head. The pack offers patients greater plaque control and whiter teeth - even if they have sensitive teeth. You will therefore be able to recommend the right brush head for each patient's needs and help them specifically tailor their brushing.

The new multi-pack contains five brush heads: the DiamondClean Standard, DiamondClean Mini, ProResults Standard, ProResults Mini and a new Sensitive Standard. The brush heads are ideal for patients with a good all round clean, more focused cleaning in specific areas or for those with sensitive teeth or tender mouths after oral surgery or because of illness.

Visit www.sonicare.co.uk/dp for more brush head information.

Reader response number 53

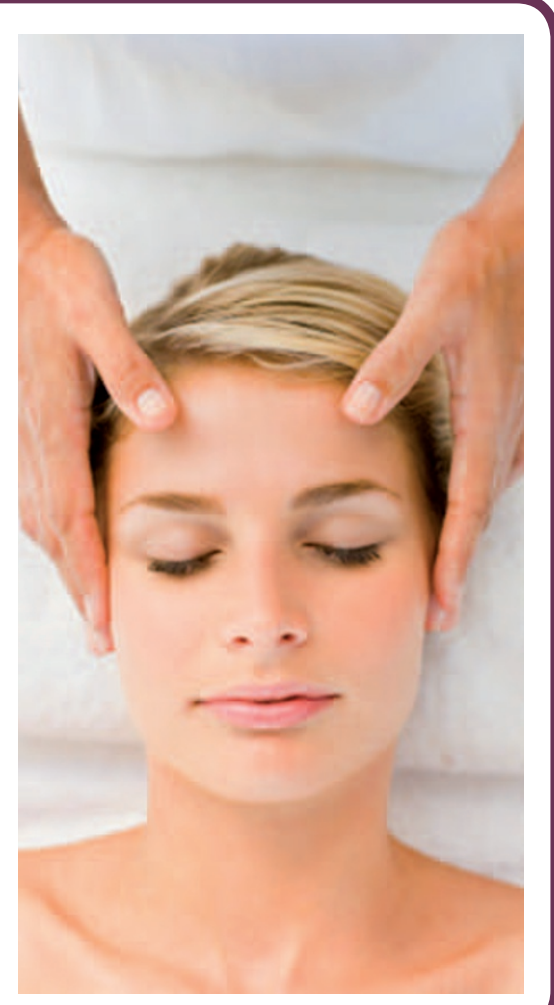

